\title{
Sens et non-sens à l'école. Violence, éducation, civilité : Pédagogie Nomade analyseur de l'institution scolaire
}

Antoine Janvier

\section{OpenEdition}

Journals

Édition électronique

URL : http://journals.openedition.org/grm/323

DOI : $10.4000 / \mathrm{grm} .323$

ISSN : 1775-3902

\section{Éditeur}

Groupe de Recherches Matérialistes

Référence électronique

Antoine Janvier, « Sens et non-sens à l'école. Violence, éducation, civilité : Pédagogie Nomade analyseur de l'institution scolaire », Cahiers du GRM [En ligne], 4 | 2013, mis en ligne le 18 décembre 2013, consulté le 30 avril 2019. URL : http://journals.openedition.org/grm/323 ; DOI : 10.4000/ grm.323

Ce document a été généré automatiquement le 30 avril 2019

(c) GRM - Association 


\title{
Sens et non-sens à l'école. Violence, éducation, civilité : Pédagogie Nomade analyseur de l'institution scolaire
}

\author{
Antoine Janvier
}

\section{Introduction}

1 En 2005, à l'initiative de l'association ardennaise Périple en la Demeure, un collectif belge d'enseignants, d'éducateurs et de chercheurs en philosophie s'est constitué en groupe de travail sur les rapports entre école et démocratie. Son nom : Pédagogie Nomade.

Ce collectif a poursuivi un double objectif. D'une part, explorer de l'intérieur les expériences scolaires qui mettent en place une pratique démocratique réelle entre professeurs et élèves (en France, au Danemark, au Rajasthan en Inde, etc.). D'autre part, rapporter et analyser ces expériences, pour les transposer au contexte particulier de la Communauté Française et y créer une école alternative.

Durant l'hiver 2006-2007, le collectif Pédagogie Nomade a rédigé un «Projet d'école différente en Communauté française ». Ce projet articulait des propositions pratiques fortement inspirées par l'expérience menée au Lycée Expérimental de Saint-Nazaire depuis 1981 à des remarques théoriques, principalement fondées sur un croisement à bien des égards improbable et paradoxal entre l'anti-pédagogie de Joseph Jacotot - d'après la lecture de la présupposition d'égalité des intelligences qu'en propose Jacques Rancière ${ }^{1}$ et la théorie institutionnelle du désir, des territoires et des devenirs de Deleuze et Guattari ${ }^{2}$. Le « Projet d'école différente » reposait sur trois axes majeurs. Premièrement, il décrétait un principe d'égalité au fondement de l'école différente envisagée : professeurs et élèves, à égalité dans l'exercice du pouvoir, décident de l'orientation et de l'organisation de l'école. Deuxièmement, il proposait d'instituer une participation de tous les membres de l'établissement à la gestion quotidienne de l'école. Troisièmement, il 
cherchait, via plusieurs options pédagogiques, à transformer le rapport des élèves et des professeurs au savoir. Après un an de négociations et de travail avec l'administration et le ministère, en septembre 2008, l'école Pédagogie Nomade ouvrait ses portes à Limerlé, près de Gouvy, en Ardenne belge, dans les bâtiments de l'association Périple en la Demeure - un vieux corps de ferme. Durant un peu plus de trois ans, dans le cadre de l'enseignement officiel de la Communauté française, Pédagogie Nomade a proposé une filière d'études expérimentales pour le cycle secondaire supérieur (équivalent du Lycée) à une douzaine d'enseignants et une soixantaine d'élèves. En novembre 2011, la ministre de l'enseignement décidait d'arrêter l'expérience sous sa forme initiale, et d'interrompre la collaboration avec l'association Périple en la Demeure ${ }^{3}$.

4 Les deux textes qui suivent ont été écrits pendant les trois années d'ouverture de l'école Pédagogie Nomade. Ils constituent à la fois un témoignage et une tentative de problématisation : problématisation de cette expérience, de ses apports, de ses difficultés, voire de ses impasses et de ses échecs; problématisation, plus largement, des questions d'éducation que Pédagogie Nomade a permis d'interroger, sinon à nouveaux frais, du moins d'un regard neuf.

5 Un dernier mot pour terminer cette introduction. La fermeture de l'école n'a pas interrompu le chemin de l'association Périple en la Demeure, entamé il y a maintenant près de 10 ans. Depuis février 2012, elle propose un lieu de vie pour ceux qu'elle appelle « les voisins », qu'un autre avait nommé «les vagabonds efficaces ». ${ }^{4}$ Son nom: la Maison Deligny 5 .

\section{Sens et non-sens à l'école}

6 On dit souvent que l'école souffre l'absence de sens. Qu'il lui faudrait retrouver des apprentissages qui aient du sens; des rapports entre professeurs et élèves qui aient du sens ; des finalités qui aient du sens. Les quelques lignes qui suivent proposent de prendre le parti inverse, et suggèrent que l'école souffre d'abord du trop de sens dont elle est investie, c'est-à-dire, comme nous allons le voir, de sa difficulté à se rapporter au nonsens qui l'affecte. On prendra Pédagogie Nomade, expérience scolaire menée entre 2008 et 2011 à Limerlé en Ardenne belge ${ }^{6}$, comme analyseur d'un certain nombre de problèmes éducatifs posés dans l'institution scolaire aujourd'hui, et surtout comme opérateur de leur déplacement et de leur reformulation.

7 Ce texte fut rédigé dans les derniers moments de l'école différente Pédagogie Nomade: il s'en ressent sans doute. On comprendra pourquoi le présent était alors de mise. Mais il faut dire maintenant qu'il l'est encore. Car Pédagogie Nomade existe toujours en Idée, c'està-dire comme problème qui donne à penser? .

8 Renouer avec le sens à l'école : c'est la nouvelle antienne dans ce monde et cette existence sans repères. Mais de quel sens parle-t-on? De celui qui donne aux élèves leur valeur marchande sur le monde du travail une fois leur éducation achevée? De celui qui fait de la vie à l'école une franche camaraderie de scouts? Ou du sens que certains voudraient voir reprendre place à l'école par-delà les affres du pédagogisme moderne destructeur de la Culture ? Le problème n'est pas de renouer avec le sens - il y en a toujours déjà, et toujours trop - mais d'avoir une petite idée, et donc une certaine prise, sur les enjeux éthiques, politiques et sociaux des sens qui «jouent» l'école, ou sur lesquels l'école se joue. 
Pédagogie Nomade ne cherche pas à renouer avec le sens à l'école. Pour deux raisons : d'abord parce qu'il serait faux d'affirmer que l'école dite « traditionnelle » soit dépourvue de sens, et que les parcours éducatifs qui s'y déroulent nagent dans l'absurde. Peut-être est-ce même d'une surcharge de sens que souffre l'école aujourd'hui, plaçant élèves et professeurs dans des situations de double contrainte, leur proposant des trajectoires et des issues contradictoires (un exemple : l'injonction faite de s'intéresser aux savoirs pour eux-mêmes ou pour les compétences qu'ils offrent; et celle qui détermine in fine le parcours d'un étudiant de les rapporter à leur valeur effective prise par l'usage qui en sera fait dans telle ou telle situation d'existence - principalement professionnelle. Et ce n'est qu'un exemple...). Ensuite et surtout parce que Pédagogie Nomade travaille en amont des tentatives qui visent à rendre un certain sens aux savoirs et aux apprentissages - là où se décident les sens de l'école, mieux : là où se décide l'évidence selon laquelle elle devrait avoir du sens.

Le rapport pédagogique qui justifie la relation entretenue par le professeur avec ses élèves, et même imposée à l'enfant dans l'institution scolaire, est en effet déjà chargé de sens. Et cela au double sens du terme : de significations et de finalités ; de partages du réel qui lui donnent du relief; de vecteurs d'orientation donnant à l'expérience un horizon, voire un but. Pédagogie Nomade propose d'interroger la consistance institutionnelle du rapport pédagogique.

11 C'est que l'on dit ennuyés ou fatigués par l'école, indifférents aux processus éducatifs, ou en porte-à-faux avec les valeurs qu'elle promeut, voire en relation d'hostilité à leur égard, un nombre croissant d'élèves. L'école, et l'éducation avec elle, seraient en crise. Sans doute. On rappellera néanmoins que le diagnostic n'est pas nouveau ${ }^{8}$. Peu importe ici la valeur historique d'un tel jugement. Il est plus intéressant pour nous de demander s'il ne s'agit pas d'un symptôme, qui nous dit quelque chose de l'éducation comme telle.

L'idée même d'une crise n'est-elle pas impliquée dans celle d'éducation, plus exactement dans l'idée selon laquelle il est nécessaire d'éduquer les hommes ?9 Car après tout, si l'éducation est jugée constitutive de l'homme, c'est bien parce qu'au départ le petit d'homme est en crise : sans ce supplément d'être qu'est l'adulte, il demeure en défaut à l'égard de ce qui lui est nécessaire pour son existence ${ }^{10}$. Thèse bien connue de la prématuration ${ }^{11}$, qui s'ancre dans une nature critique - au sens où l'on parle de situation critique - de l'homme comme tel, par laquelle il doit donner du sens à ce qu'il vit et à ce qu'il est - découper un ordre, valoriser le réel, poser des fins. Le sens ne lui est pas donné tout fait dans une expérience déjà réglée et achevée, ou par des instincts grâce auxquels il se le donnerait pour ainsi dire «naturellement ». L'éducation trouve sa place dans la vie humaine comme le cadre par lequel les individus deviennent des êtres humains et conquièrent une autonomie par conséquent toujours relative, puisqu'ordonnée aux finalités dans lesquelles tout «nouvel arrivant » s'insère pour cette raison même qu'il est en défaut sur sa propre expérience.

13 Les variantes de ce schème fondamental, le schème pédagogique, peuvent être aussi différentes que possibles et se combiner de toutes les manières : modèle autoritaire fondé sur la structure de sens à laquelle il faut que l'individu se plie pour se constituer en sujet, et ensuite (mais seulement ensuite) pour être lui-même partie prenante de la structure ; modèle médical du soin nécessaire à ces êtres sans défense (pas de poils, pas d'écailles, pas de griffes ou de crocs), qu'il s'agit de protéger ; modèle pastoral du troupeau perdu qui a besoin de guides pour initier son orientation; modèle psychologisant qui appuie l'acte éducatif sur une compréhension première des besoins et des désirs, de l'imaginaire 
et des nécessités de l'enfant pour mieux construire son parcours propre; modèle naturalisant qui appuie le rapport éducatif sur les activités « naturelles » ou élémentaires des enfants à partir desquelles seul leur intérêt est mobilisable et peut en conséquence être dévié vers les étapes futures de l'apprentissage, nécessaires à leur réalisation en êtres majeurs. Quelles qu'elles soient, ces variantes restent inscrites dans le spectre ouvert par le schème pédagogique, qui marque les uns de dépendance à l'égard des autres, comme condition même de leur future indépendance relative.

Mais voilà, ce qui justifie l'institution de cette relation pédagogique est aussi ce qui la menace d'échec et, parfois (de plus en plus souvent ?) d'impossibilité. La situation critique première n'est pas supprimée par l'éducateur : elle est travaillée, conjurée, repoussée, refoulée même; elle peut donc toujours faire retour. Le sens construit dans l'éducation n'est jamais garanti - c'est justement ce qui le justifie du point de vue du schème pédagogique. Un écart de l'élève par rapport aux ordres de significations et de finalités dans lesquels il est inscrit est toujours possible ; mieux, c'est précisément le sens de l'acte éducatif lui-même que de le rendre possible dans certaines limites, permettant ainsi la conversion de l'élément critique en énergie de transformation de la structure. Ce qu'on appelle crise de l'éducation, c'est moins son démantèlement extérieur ou la déchéance de son organisation que cet effet ou ce contre-effet paradoxal du schème pédagogique donateur de sens.

Que signifie un tel retour du refoulé, pour parler comme Freud ? Qu'il n'y a pas de raison fondamentale - assurée - au parcours éducatif, puisqu'il se justifie de la possibilité de sa propre absence (c'est parce qu'il n'y en a pas qu'il en faut). Seule est fondée la contingence de l'existence et des rapports qui la composent, autrement dit : le non-sens (l'in-fondé) de ce fait qu'on existe. Mais il faut faire un pas de plus. Car c'est la finalité de l'éducation elle-même qui prête le flanc à ce retour du non-sens sans lequel le sens ne serait pas : c'est le monde de l'adulte qui se trouve en réalité affecté par la mise en crise de l'éducation. Et c'est d'abord son propre ordre de significations et ses propres finalités qui sont ainsi suspendus, rendus à leur contingence première, c'est-à-dire soustraits à l'évidence que l'on s'efforçait d'y voir. Avant de vouloir rendre du sens à une école et à un processus ou à un rapport qui en aurait perdu, on peut se demander si le remède n'est pas aussi la cause de ce qu'il est censé guérir. Il apparaît en effet que le sens et le non-sens sont contemporains et en relation de présupposition réciproque, de telle sorte qu'à surenchérir sur la nécessité du premier, l'on rend plus éclatante la présence du second, qui insiste et produit ses effets à la mesure où l'effort pour le conjurer se fait impérieux ${ }^{12}$. Qu'il y ait une multiplicité de sens à l'œuvre dans ce qu'on appelle l'école (terme qui recouvre pour cette raison plusieurs réalités: plusieurs manières de découper et de mettre en ordre le réel, et plusieurs parcours constitués) tient sans doute à la nécessité, sinon de repousser, au moins de médiatiser le rapport des sujets au non-sens de leur contingence, de le rendre vivable et de se donner une prise sur lui. Mais que cette multiplicité aille croissant, qu'elle durcisse et se segmente au point de ranger certains dans ce que Maud Mannoni appelait un « destin institutionnel $»^{13}$, ou qu'elle recouvre des double-binds perturbants pour n'importe qui, tient peut-être à ce que l'on n'accepte de voir le non-sens qui l'affecte et l'affectera toujours que comme un élément perturbateur à supprimer (formule abstraite qui s'instancie dans certaines figures déterminées et actions concrètes : celui qui dérange et qui n'est pas à sa place, l'acte qui conteste et désordonne), produisant ainsi le contraire de ce qui était souhaité. 
16 La fameuse idée d'égalité présupposée par Pédagogie Nomade comme le point d'interrogation ou de problématisation des rapports profs/élèves n'est pas née du désir de réaliser la démocratie à l'école, ni de la volonté charitable de considérer de pauvres étudiants pourtant plutôt sympas et intelligents comme des égaux, mais de la nécessité éprouvée par quelques uns de sortir du double mouvement d'oscillation contradictoire entre un trop plein de sens imposés (et parfois choisis, suivis, repris) et le non-sens d'une situation précisément saturée de significations et de finalités: ce qui, pour aboutir, demandait que chacun, dans l'institution, trouve les lieux et les moments où il peut suspendre, interroger, et se positionner par rapport aux différents sens qui « le jouent », c'est-à-dire se soustraire à l'injonction qui lui est faite d'être d'un côté ou de l'autre de la ligne de partage entre le majeur et le mineur, et sente ou éprouve sa propre capacité à avoir prise, à sa manière, sur la situation, ses déterminants et ses issues.

17 Dans une structure qui reste une structure scolaire, soumise à ses prescrits légaux (ceuxlà qui sont contrôlés par l'inspection, pour le pédagogique, et par l'administration, pour l'institutionnel) et à ses mythes fondateurs officiels (par exemple le célèbre Décret «Missions »), cette tentative de faire face au non-sens pour y mettre à l'épreuve les différents sens de l'école risque toujours d'être repliée sur certains d'entre eux, portés par les enseignants eux-mêmes comme moteur de la singularité de leur acte éducatif : autorité brute coupée de toute intention d'apprendre autre chose que le pur rapport d'inégalité qui différencie le maître de l'élève (lui épargnant le recouvrement du rapport par un motif illusoire et porteur, en réalité, d'exploitation : l'apprentissage des savoirs, de la vie en société ou de la discipline) ; pitié caritative pour les « exclus » du système qui optent en dernier recours pour cette école (cette morale qui conditionne l'éducation au rapport amical, voire amoureux - sublimé s'entend); affection socio-politique des professeurs engagés pour ces gamins-qui-ont-osé-y-aller (s'identifiant imaginairement à l'élève, au risque de détruire tout pas d'écart permettant aux uns et aux autres de sortir de la relation et donc de conquérir une forme d'autonomie); position du guide venant en aide aux désorientés ; éducateur thérapeute soignant les blessures des exclus, etc. ${ }^{14}$

Pédagogie Nomade ne présente pas de remède miracle contre ces retours de la structure scolaire plus ou moins refoulés dans sa mise en question elle-même - retours du refoulé propres aux expérimentations : c'est ici l'institution scolaire qui est en partie refoulée par la tentative d'une école différente. Pédagogie Nomade en joue sans doute. Précisément, tout repose sur ce «jeu». Il ne s'agit pas de proposer une expérience hors des finalités et significations qui structurent le monde scolaire, mais de s'écarter de leur évidence : on cherche à « décoller » le rôle joué par l'un ou l'autre de sa propre personne, et à le faire circuler parmi le collectif qui compose l'institution, professeurs comme élèves ; et à partir de là à créer les temporalités et les espaces pour que dans ce bouleversement de l'identification rôle/personne, puisse se dire et se mettre en jeu le rapport à la contingence de la présence des uns et des autres dans l'institution scolaire; enfin, en s'appuyant sur des temps collectifs (comme les conseils de progrès) ou plus intersubjectifs (comme les groupes de suivis), à apporter des pistes de solutions aux problèmes posés par l'affrontement du rapport au non-sens qui affecte les sujets et leurs positions respectives. L'ensemble de ces dispositifs n'ayant de chance de fonctionner qu'à la condition supplémentaire d'être eux-mêmes appréhendés depuis leur propre contingence, et donc subordonnés à la règle que « ce qui se passe » dans le règlement du rapport au non-sens peut toujours se faire ailleurs que là où c'était prévu, anticipé, formalisé. 
C'est pourquoi Pédagogie Nomade se heurte sans cesse à sa propre impuissance : car c'est bien l'impuissance des sens de toute structure scolaire qu'elle se met en demeure d'affronter et de traiter. Tout est affaire d'analyse prudente et précise, au sens que la psychanalyse donne à ce terme : moins une réflexion discursive sur ses actes et son passé qu'un retour sur soi qui est transformation de son rapport aux autres, au monde et à soimême. Pédagogie Nomade s'efforce de déplacer l'institution-école de son point critique à ce point analytique - en ce sens, elle est autant une machine à « déranger le système » qu'un lieu d'expérimentation de solutions à ses problèmes et de pistes de transformations profondes. Il est temps qu'elle soit perçue comme telle. Car les disfonctionnements dudit système ne laisseront guère longtemps l'illusion qu'il existe réellement comme tel.

\section{Violence, éducation, civilité : Pédagogie Nomade analyseur de l'institution scolaire}

20 Depuis les Lumières, qui donnent le mythe de nos idéaux éducatifs, l'éducation a pour but, à travers l'apprentissage de certains savoirs, l'institution d'un rapport à soi et d'un rapport aux autres placée sous le signe du contrôle de soi (ou de la discipline) et de la civilité, c'est-à-dire la domestication, voire la suppression de la violence qui menace de destruction les sujets et les rapports sociaux. L'école trouve là une de ses missions : lieu de culture dans les deux sens du terme - acquérir une ou « de la » culture, mais aussi se cultiver, passer de l'état de nature à l'état de culture. Par définition, la violence n'a rien à y faire ; elle en est exclue. Ce qu'on appelle la « crise de l'éducation » ou la crise de l'école, c'est bien souvent l'échec de cet idéal, qui se manifeste à la fois dans les phénomènes de violence au sein de l'école (réels pour une part, mais fantasmés pour une part également) et, surtout, dans l'impossibilité aujourd'hui de maintenir cachée l'exclusion concrète de ceux-là qui sont considérés comme les « causes » de cette violence.

21 J'aimerais montrer que Pédagogie Nomade, expérimentation scolaire en Communauté française fondée sur l'idée d'égalité entre professeurs et élèves et appuyée sur un principe de cogestion ${ }^{15}$, prend à rebours le mythe moderne de l'éducation, et tente ainsi de reformuler les problèmes posés par la violence - plus, sans doute, que d'apporter une solution. Plutôt que d'essayer d'éliminer la violence, il s'agit d'en chercher collectivement les causes; plutôt que de la refouler en la méconnaissant, s'exposant à des retours d'autant plus durs qu'ils sont fantasmés impossibles en droit (« ne devant pas se produire à l'école »), il s'agit d'en faire un problème interne à l'institution, et donc de chercher, dans les limites qui sont les siennes, les moyens de transformer l'institution elle-même. Pédagogie Nomade ne propose pas une voie d'éducation qui réalise enfin l'idéal de nonviolence inscrit dans le concept de citoyen qui structure la scolarité moderne. Cette école différente s'efforce de transformer regards et pratiques sur les violences, visant deux objectifs distincts et complémentaires : $y$ voir autre chose que de la seule violence, et se donner quelque prise sur ses effets ${ }^{16}$.

Mon propos se situe sur un double plan : un plan idéel et un plan réel. Il concerne bien le mythe de l'éducation, mais aussi son lieu privilégié de prolifération et de réalisation, l'école, l'institution scolaire. Plus exactement, je tenterai de me situer à l'articulation de ces deux plans, là où ils se croisent : là où l'institution scolaire conditionne et permet le développement du mythe de l'éducation ${ }^{17}$; là où, en retour, le mythe de l'éducation produit ses effets sur ou dans l'appareil scolaire, là où il se réalise si l'on veut, mais à la 
condition de voir dans cette réalisation autre chose que la simple reproduction dans le réel de ce que nous dit le mythe.

Que nous dit ce mythe? Un mot d'abord sur l'expression. Par mythe de l'éducation, il ne faut pas entendre un idéal éducatif parmi d'autres, une voie possible d'éducation. Si je parle de mythe de l'éducation, c'est pour renvoyer à ce que l'idée même d'éducation enveloppe comme représentations et valorisations (ou normativités) du rapport à soi, au monde et aux autres.

Idéalement l'éducation est domestication de la satisfaction de ses pulsions individuelles et apprentissage de la socialisation : institution d'un rapport à soi et d'un rapport aux autres et au monde éliminant la violence destructurante de pulsions non-maîtrisées. De ce point de vue, l'éducation repose sur l'idée d'une contre-violence imposée à la violence première de l'immaîtrisé pulsionnel - c'est tout le thème du dressage, retraduit dans celui de la discipline ${ }^{18}$.

Passons au plan réel. L'institution scolaire s'est construite comme le lieu de l'éducation par excellence ou privilégié (contrairement à la famille par exemple, qui se trouve remplir une fonction éducative de fait, et parmi d'autres) : comme lieu de réalisation du mythe; comme lieu où ce mythe se réalise en fonction des rapports sociaux déterminés dans lesquels cette réalisation s'insère. Lieu de la discipline et de la maîtrise de soi, en même temps, mieux, par le biais des savoirs maitrisés, des disciplines apprises. Mais - et c'est pour ça que je parle de mythe - cette réalisation de l'éducation s'est toujours heurtée à un échec plus ou moins relatif, qu'elle a géré ou traité de manières différentes, comme autant de points critiques susceptibles de basculer en autant de situations de crises, c'està-dire mettant en crise l'idée même d'éducation (non pas seulement sa réalisation, mais son idéalité). Or il est possible que ce qu'on appelle aujourd'hui «crise » de l'éducation soit l'échec de l'institution scolaire à affronter ses points critiques, c'est-à-dire ses échecs : non pas donc que ce qui auparavant marchait ne marche plus, non pas que nous soyons entrés en décadence après avoir connu un âge d'or où l'école produisait de bons éléments, où les maîtres étaient écoutés et respectés, etc. ; plutôt une sorte de retour de ce qui était déjà des échecs, mais que l'école avait réussi à refouler par un ensemble de stratégies permettant à ses échecs de n'être pas les siens - c'est-à-dire lui permettant de croire et de faire croire, de jouer et de faire jouer son propre idéal.

Comment l'école gère-t-elle ses propres échecs? Par la présence de discours et de pratiques disciplinaires ou de moralisation (il ne faut pas, on ne peut pas, etc.), éliminant par avance ce qu'elle est pourtant censée éduquer, ce sur quoi elle est censée s'appliquer (ce qui conduit à ce paradoxe que, pour aller à l'école, il faut être déjà éduqué); par la mise en concurrence interne des élèves dans le procès d'acquisition des savoirs, qui consiste en une individualisation des «capacités » et qualités des uns et des autres, telle que les obstacles à l'éducation peuvent être extériorisés par l'institution scolaire, en les psychologisant ou sociologisant, l'impuissance à éduquer certains apparaissant rétrospectivement comme une limite naturelle révélée par l'œuvre éducative elle-même dans la compétition pédagogique (les points, classements, prix); par des processus plus ou moins discrets de réorientations, c'est-à-dire de différenciations et de segmentations, soit dans l'institution scolaire elle-même (celui-là est bon pour aller en technique, etc.) soit en dehors (par exemple sous la forme de l'insuffisance psychologique - écoles spéciales voire institution psychiatriques - ou de l'inadaptation sociale - des institutions de redressement aux centres de rééducation); par des procédés de sélections, là encore plus ou moins habiles, qui en même temps qu'ils permettent à certains de "poursuivre " 
leurs études l'interdisent à d'autres et les envoient hors de l'école ; enfin, par l'exclusion, qu'elle soit attribuée à la responsabilité de l'enfant ou de sa famille (se mettant devant le fait de la déscolarisation) ou qu'elle soit assumée voire revendiquée par l'école elle-même, envoyant directement l'élève sur le chemin du travail ou le laissant aux prises avec d'autres institutions, scolaires ou non, ce qui l'engage dans un parcours se terminant dans le meilleur des cas par son introduction dans le sacro-saint monde du travail - et bien trop souvent dans ses marges, voire en son dehors ${ }^{19}$.

Ce qui est frappant aujourd'hui, ce n'est pas que toutes ces stratégies de gestion de l'échec par sa différenciation et sa répartition sur des voies qui l'empêchent d'éclater comme tel, n'existent plus ; c'est que leur fonctionnement ne suffit plus à faire fonctionner de l'autre côté l'idée même d'éducation: autrement dit ces différentes stratégies ne nous apparaissent plus comme des manières d'éduquer, comportant toujours un envers positif d'émancipation (ou plutôt un endroit dont elles seraient seulement l'envers), d'acquisition des savoirs, de construction de soi et de socialisation, c'est-à-dire en somme de refoulement, suppression ou sublimation de la violence et d'ouverture d'un espace où l'on peut construire une existence, une existence qui dure. Ces stratégies ne nous apparaissent plus que comme des stratégies d'échec, et donc échouent elles-mêmes, à leur tour, en tant qu'elles ne sont plus des moyens de réalisation de l'éducation. Elles fusionnent, en quelque sorte, pour n'être plus que des aveux d'échec, ce qui se traduit par le fait qu'elles ne sont plus comprises que depuis leur cas le plus extrême: autant de manières d'exclure.

C'est pourquoi, de l'autre côté, elles mettent en échec l'idée même d'éducation comme processus de sublimation ou de conversion de la violence pulsionnelle : car la réalisation de ce processus non seulement apparait de plus ou en plus comme l'exercice d'une contre-violence sans effets transformateurs (sublimants), mais surtout cet exercice se montre lui-même producteur de violences nouvelles illocalisables. Ceci des deux côtés de la barrière : du côté des élèves ou des «mineurs »; du côté des adultes, professeurs ou majeurs.

Pédagogie Nomade s'inscrit dans ce contexte. Je pense qu'on peut en comprendre l'expérience comme une tentative de réactiver différentes stratégies de gestion/ traitement des échecs de l'éducation, mais d'une autre manière que celles qui furent proposées naguère par l'institution scolaire et que celle-ci continue de proposer aujourd'hui ; et c'est cette autre manière qui conduit de fait Pédagogie Nomade à remettre profondément en cause le mythe de l'éducation et donc son idée même.

Que fait-on à Pédagogie Nomade? D'abord instaurer les conditions d'un rapport qui permette le traitement de la violence autrement que sous la forme de sa dénégation, autrement que sous la forme traditionnelle d'une contreviolence opérateur de conversion.

31 Sur quoi se trouve fondée cette forme? Sur l'idée d'un point terminal qui offre la fin du mouvement éducatif déterminant le juste comportement (le juste rapport à soi et aux autres) et la manière même dont il faut y accéder. Et ceci est intégré dans l'idée d'un juste rapport au savoir : le juste rapport à soi et aux autres est donné comme la seule condition d'un juste rapport au savoir, comme la bonne manière dont on peut apprendre (on n'apprend pas debout, dans le bruit, etc.) C'est donc en même temps que le bon comportement le critère de ce qu'il faut apprendre et de la manière dont il faut l'apprendre qui est le but de l'opération de conversion de la violence (c'est par là qu'il faut passer, telle et telle étape, dans tel ordre et selon telle manière ; c'est ça qu'il faut 
connaître et pas autre chose, parce que c'est ça qui fait partie du socle commun ou des compétences terminales communes par lesquelles l'intégration au monde social est possible). Ce point de finalisation de l'éducation comme étant en même temps un certain apprentissage déterminé est instancié dans une figure : le maître - celui qui se maîtrise et qui maîtrise les savoirs. L'école tout entière est fondée sur ce rapport dissymétrique, qui passe moins entre ceux qui savent et ceux qui ne savent pas, entre savants et ignorants (bien que le rapport se nourrisse de cette différence: mais elle ne le définit pas en propre), qu'entre quelque chose comme des capables et des incapables qui sont aussi des autonomes et des dépendants, des achevés et des inachevés, en somme des majeurs et des mineurs.

Le geste premier de Pédagogie Nomade est de transformer cette dissymétrie au profit d'un rapport d'égalité entre élèves et professeurs. Cette transformation s'est appuyée sur la lecture du livre de Jacques Rancière, Le maitre ignorant ${ }^{20}$. L'expérience de Jacotot débouche sur ce constat surprenant : on peut enseigner ce qu'on ne sait pas. D'où la thèse ou plutôt l'hypothèse d'une égalité des intelligences qui va à l'encontre du principe même de la pédagogie, selon lequel le maître est nécessaire, non seulement en vertu de son savoir, mais en vertu du savoir sur ce savoir : du savoir de la manière dont ce savoir doit être appris et enseigné, du savoir du parcours adéquat, du chemin à faire pour acquérir ce savoir.

Mais ni Jacotot ni le livre de Rancière ne s'en tiennent à cette découverte. La leçon enveloppée dans l'idée du maître ignorant, c'est celle d'un apprentissage qui se fait toujours à l'insu du maître, ce qui signifie que non seulement il ne sait pas ce qu'il apprend, non seulement il ne sait pas comment l'apprendre, mais par conséquent il ne sait même pas quelle est sa place de maître, et ne maîtrise donc pas la finalité de l'apprentissage. Bien sûr je pousse à la limite la logique du maître ignorant; car dans le livre, il apparaît bien que le père analphabète, s'il ne sait ni lire ni écrire et donc pas plus comment apprendre à lire et à écrire, sait néanmoins que son fils cherche à lire et à écrire, et c'est parce que l'un et l'autre savent ce qu'ils sont en train de faire et qu'ils savent dans quel but ils le font que le père peut vérifier qu'il y a bien apprentissage.

Or cela suppose déjà que l'un et l'autre veulent apprendre ce qu'ils veulent apprendre, et le savent. Mieux, cela suppose que l'élève est déjà en chemin, et que c'est lui qui va chercher son maître, plutôt que l'inverse. Le maître ignorant nous révèle ceci : il n'y a pas d'apprentissage forcé ou imposé, ou plutôt il n'y en a que si l'élève est déjà engagé dans la démarche d'apprentissage, de telle sorte que la contrainte ne lui apparait pas comme une pure force extérieure dont il subirait les effets, mais est ressaisie par lui comme correspondant avec son propre désir d'apprendre, et vécue comme légitime et nécessaire à la réalisation de sa propre volonté. On peut dire les choses plus simplement et avec un peu plus de précision : il y a circularité entre la mobilisation par l'élève de sa volonté d'apprendre et la contrainte pédagogique imposée par le maître, de telle sorte que la contrainte n'est jamais purement et simplement cause de l'apprentissage, mais ne produit d'effet que sous la présupposition qu'elle correspond à la volonté d'apprendre, autrement dit sous la condition de remplir une case essentielle ou nécessaire de la structure de réalisation du désir d'apprentissage.

C'est pourquoi le maître ignorant constitue une critique en règle du rapport pédagogique, c'est-à-dire de l'idée même d'éducation: de l'idée selon laquelle ce qui marche dans le rapport éducatif est dû à ce rapport lui-même ; de l'idée selon laquelle la transformation subjective de l'élève est causée par la contrainte (plus ou moins contraignante) du maître. 
Si le maitre est ignorant, il l'est avant tout de ce en quoi il est maitre, de ce par quoi il est maitre, et je dirais même, bien souvent: de ce pour quoi, en vue de quoi il est maitre. L'égalité des intelligences prend alors un sens supplémentaire. Ce n'est pas seulement le présupposé selon lequel tout ce qu'une intelligence humaine a produit ou compris, n'importe quelle autre intelligence humaine le peut également. C'est aussi et surtout une manière de nier la dissymétrie de principe entre majeur et mineur, qui structure le rapport éducatif: cette dissymétrie selon laquelle certains savent où et comment entraîner sur le chemin de la construction subjective d'autres qui ne le savent pas. L'égalité des intelligences ne fait pas seulement effet sur la confiance en soi de l'élève (je peux comprendre ce qu'a pu comprendre n'importe quel autre). Elle fait effet sur le maître lui-même : elle lui soustrait la certitude selon laquelle il détient la fin et partant les moyens de l'éducation; elle rompt l'évidence du partage mineur/majeur, parce qu'elle retire au maître ce qui fait de lui un maître, à savoir la maîtrise du processus éducatif, son contrôle des modalités et des finalités de l'apprentissage. L'égalité des intelligences, c'est la fin de l'inégalité des capacités, et avant tout de celle qui sépare les capacités de ceux qui savent comment user de leurs capacités et ceux qui ne le savent pas et auraient besoin, en supplément de leurs capacités, de la capacité de savoir s'en servir à bon escient, sur les bons objets, selon les bonnes manières, pour le bon objectif. En somme, l'égalité des intelligences affecte les rapports de pouvoir, plus exactement la dissymétrie inégalitaire des rapports de pouvoir qui structure la relation éducative et détermine les fins de l'éducation.

36 C'est ici que le maître ignorant rejoint notre problème de départ: celui de la violence dans le rapport éducatif. C'est que la contre-violence n'apparaît pas nécessairement comme force physique exercée sur l'élève, ni même comme force symbolique. Elle désigne d'abord l'univocité de la décision pédagogique, c'est-à-dire ceci que le maitre décide, et que le rapport de pouvoir joue, en droit, en un seul sens, sans réciprocité. Ainsi le rapport peut, peut-être, s'inverser : la contre-violence éducative se joue avant toute contrainte dans le geste qui distingue des élèves et des maîtres, des mineurs et des majeurs, et dans l'attribution, corrélative de ce geste, de la décision des fins et des modalités du parcours pédagogique, à un seul des deux côtés, mais aussi et pour cette raison, dans l'attribution aux seuls maîtres de la capacité à décider quand la barrière est annulée et a été franchie.

Ce qui m'intéresse ici, c'est qu'il apparait que le rapport éducatif se justifie de ce qu'il impose. Il légitime ce qu'il crée en le créant : il légitime la contre-violence par la nécessité de contraindre, d'orienter, de permettre de structurer une violence première qui, laissée à elle-même, conduirait l'élève à sa destruction, voire à celle des autres ; mais l'idée même que cette violence première nécessite pour être canalisée, régulée, convertie et sublimée, une contre-violence issue du maître, repose d'abord sur l'instauration de la coupure entre élève et maître, enfance et adulte, mineur et majeur. Peut-être est-ce là la violence primaire, dans la présupposition qu'il y a d'un côté de la violence primaire et de l'autre une contre-violence légitime parce que seule dotée de la capacité de conversion. D'où la question : ce que certains pointent comme des retours de barbarie dus à la défaite de la culture et de la civilisation dans les manifestations d'agressivité envers les murs de l'école, ou l'autorité des enseignants, ne doit-il pas être considéré aussi, au moins pour une partie, comme des tentatives de redonner du jeu, c'est-à-dire de la réciprocité conjurant ainsi une situation intenable pour certains qui se vivent dépossédés de tout pouvoir, c'est-à-dire impuissants? Et cela vaudrait à l'inverse, dans certaines réactions 
agressives des enseignants envers les élèves, qui sortent du rôle de contre-violence légitime que leur attribue l'institution scolaire ?21

En prenant pour présupposition l'égalité entre professeurs et élèves comme cas d'exercice de l'égalité des intelligences - aussi bien dans les décisions qui concernent la vie de l'école que dans leur mise en application et dans la gestion quotidienne, ainsi que, pour une bonne part, dans les processus d'apprentissage -, Pédagogie Nomade ne vise pas seulement, comme il fut posé initialement, à rendre le goût du savoir et le désir d'apprendre à des élèves qui en étaient progressivement dépourvus sous l'effet d'un enseignement traditionnel qui ne leur convenait pas. Plus profondément, il s'agit de réintroduire du politique à l'école, et de le réintroduire par le biais d'une interrogation sur les rapports de pouvoir. Cette interrogation m'apparait, au moins partiellement, comme une interrogation sur la violence, comprise comme ce point limite où le rapport de pouvoir porté à l'extrême en vient à s'annuler. Là où face aux phénomènes d'agressivité plus ou moins intenses, qu'ils soient symboliques ou physiques, l'école oppose traditionnellement une contre-violence légitimée par l'hypothèse de ses effets de conversion, Pédagogie Nomade s'efforce de suspendre le partage déterminant le rapport agressivité/discipline pour, appuyé sur l'égalité de pouvoir entre élèves et professeurs, en faire un problème collectif qui concerne au même titre les uns et les autres en tant qu'ils participent d'un même collectif. Cela veut dire que, potentiellement, ce qui était codé ou compris comme agressivité se trouve réarticulé en sa dimension politique comme la tentative plus ou moins désespérée, plus ou moins radicale, avec des effets plus ou moins contrôlables, de rejouer un rapport de force et de réintroduire de la réciprocité là où le rapport avait tendance à aller à sens unique, c'est-à-dire à n'être même pas un rapport.

C'est pourquoi Pédagogie Nomade est un lieu éminemment paradoxal où se réfléchissent parfois avec fracas des violences qui, d'autre part, se trouvent par là réinterprétées ou relues autrement, traduites politiquement, ou plutôt : des phénomènes d'agressivité qui sont simultanément l'objet d'une reproblématisation politique, mettant en question les rapports de pouvoir au sein de l'école. Disons les choses un peu autrement. En plaçant au cœur de l'école l'égalité entre professeurs et élèves, il s'agissait de court-circuiter d'emblée la situation d'opposition entre violence et contre-violence sans issue autre que la conversion au cours du processus éducatif (c'est-à-dire la subordination des forces en jeu à la force légitime de l'école représentée par le corps enseignant et éducateur) ou son extériorisation hors de l'école comme un problème qui n'est pas de son ressort, jusqu'à l'extériorisation absolue hors de l'institution. C'est le sens à Pédagogie Nomade d'un ensemble d'espaces où peut se construire le conflit, où les puissances contradictoires des uns et des autres peuvent trouver les moyens de coexister : l'assemblée générale bien sûr, mais aussi les groupes de suivi, le dispositif de co-évaluation, le conseil de progrès, etc. ; mais aussi, plus important je crois, on trouve à Pédagogie Nomade, autant qu'une politisation du pédagogique, une transformation du politique en fonction d'enjeux pédagogiques, au sens où, autant que les partages qui structurent l'organisation des apprentissages sont posés comme enjeux politiques, les conflits et problèmes de pouvoirs sont posés comme des occasions d'apprendre.

Du coup le conflit prend un tout autre sens, puisqu'il est considéré comme nécessaire, pièce interne du processus scolaire et réarticulé directement aux savoirs. Cela peut avoir pour conséquence qu'à Pédagogie Nomade il y a moins de violence qu'ailleurs. Mais je ne suis pas sûr que ce soit tout à fait vrai, ni que ce soit la meilleure manière d'aborder le problème : c'est plutôt que la violence y est autrement traitée, comme faisant partie des 
rapports de pouvoir nécessairement à l'œuvre dans des rapports sociaux ou interindividuels, a fortiori dans l'institution scolaire où ils sont censés fonctionner à sens unique (contrairement aux rapports sociaux entre « adultes » ou majeurs) et donc, in fine, ne pas exister, annulés dans l'évidence d'une dissymétrie dessinée par la place du maître. D'où le fait qu'en un sens il est juste de dire qu'il y a moins de violence à Pédagogie Nomade : c'est-à-dire qu'il y a moins de violence faite aux professeurs, sans doute, moins d'agressivité envers l'équipe d'enseignants, et plus largement envers l'école elle-même, puisque la place occupée par les uns et par les autres est différente et que les uns comme les autres se trouvent en position relative d'exercer les différents pouvoirs qui parcourent l'institution, et donc par là aussi une certaine agressivité. Mais on doit ajouter d'emblée que cette violence, en même temps, circule et se répartit autrement : elle ne se décharge pas, ou moins, par à coups, et en sens uniques (quel que soit le sens).

41 Pédagogie Nomade ne résout donc pas le problème de la violence à l'école. Bien au contraire, on pourrait dire qu'elle pratique à son égard la surenchère pour le déplacer et le transformer. Mais n'est-ce pas un nouveau mythe de l'éducation, une nouvelle forme de conversion de la violence et de sublimation, qu'on dirait politique, par opposition au mythe moral ou civilisateur? Peut-être ; en un certain sens, oui. Mais en un certain sens seulement. Car l'instauration de la conflictualité au centre de l'école soustrait ce qu'il s'y passe à l'idéal d'une amélioration des uns et des autres qui progressivement apprendraient à passer de la violence au conflit. Cela très concrètement signifie que font partie de la vie de l'école les surgissements de violence au sein même des rapports conflictuels ou en dehors : le conflit est moins un état que le travail même de la violence. Cela veut dire aussi que Pédagogie Nomade flirte sans cesse avec un double écueil (mais c'est sans doute l'écueil même dans lequel oscille l'école, et que Pédagogie Nomade, comme un miroir grossissant, se risque à réfléchir et à agrandir) : les retours brutaux de violence de part et d'autre (ras-le-bol plus ou moins violemment, justement, exprimés); la désaffection ou l'indifférence, un certain épuisement à l'égard du travail incessant à faire - autrement dit deux formes de réaction sans réciprocité manifestant l'insupportable des tensions inhérentes au travail de la violence.

Mais justement parce que ces deux écueils sont considérés comme des menaces immanentes et permanentes de l'école, il me semble qu'il ne s'agit pas là d'une réitération du mythe éducatif. Pédagogie Nomade, c'est peut-être plutôt une contre-école.

\section{NOTES}

1. Voir J. Rancière, Le maître ignorant, Paris, Fayard, 1987, rééd. 10/18, 2004. Jacques Rancière apportera son soutien au projet et à l'école Pédagogie Nomade: http:// www.peripleenlademeure.com/spip.php?article47.

2. Voir G. Deleuze et F. Guattari, L'anti-〔Edipe, Paris, Minuit, 1972. Et Mille Plateaux, Paris, Minuit, 1980. 
3. On trouvera le récit des péripéties qui jalonnèrent le long processus de fermeture ici : http:// www.peripleenlademeure.com/spip.php?article288. Et quelques prises de position et réflexions à ce sujet à l'adresse suivante : http://www.peripleenlademeure.com/spip.php?rubrique17.

4. F. Deligny, Les vagabonds efficaces, Paris, Michalon, 1947, rééd. dans F. Deligny, Euvres, éd. S. Alvarez de Toledo, 2007.

5. On en trouve les Parti-pris pédagogiques sur le site de l'association: http:// www.peripleenlademeure.com/spip.php?article356.

6. Plus d'informations au sujet de cette école sur le site www.peripleenlademeure.be, section «Pédagogie Nomade».

7. Voir G. Deleuze, Différence et répétition, Paris, PUF, 1968. Sur l'articulation entre Idée et problème, voir en particulier le chapitre 4.

8. Voir par exemple le texte célèbre d'H. Arendt, «La crise de l'éducation » (1961), dans La crise de la culture, Paris, Gallimard, 1989. On pourrait remonter bien plus loin.

9. Au sens où l'entendait Kant : «L'homme est la seule créature qui doive être éduquée. » (I. Kant, Réflexions sur l'éducation, tr. A. Philonenko, Paris, Vrin, 1967, rééd. 2004). Formule à rapporter à la maxime célèbre: "L'homme est un animal qui, lorsqu'il vit parmi d'autres individus de son espèce, a besoin d'un maître ». Ce qui, il convient de le rappeler, fait fondamentalement problème selon Kant. Car si l'homme "a donc besoin d'un maître qui brise sa volonté particulière et le force à obéir à une volonté universellement valable, afin que chacun puisse être libre ", " où prend-il ce maître? ", sinon chez un autre homme qui, en tant qu'homme, a tout autant besoin d'un maitre : « Mais ce maître est, tout comme lui, un animal qui a besoin d'un maître » (I. Kant, "Idée d'une histoire universelle au point de vue cosmopolitique » (1784), $6^{\text {ème }}$ proposition, trad. L. Ferry, dans I. Kant, CEuvres philosophiques, II, éd. F. Alquié, Paris, Gallimard, La Pléiade, 1985, p. 195). Ce paradoxe sera repris, bien qu'en d'autres termes et dans un autre contexte intellectuel et conceptuel, dans la troisième des onze Thèses sur Feuerbach de Marx. Il constituera l'un des leviers de la critique portée par Jacques Rancière sur le rapport inégalitaire qu'entretiennent certains intellectuels communistes à l'égard du prolétariat (voir J. Rancière, La leçon d'Althusser, Paris, Gallimard, 1973). On peut y voir, en creux, une première esquisse des développements proposés plus de dix ans plus tard sur la présupposition de l'égalité des intelligences dans Le maître ignorant (Paris, Fayard, 1987, rééd. 10/18, 2004).

10. Voir à ce sujet les premières pages percutantes et suggestives de R. Schérer, Émile perverti, Paris, Robert Laffont, 1974, rééd. Désordres/Laurence Viallet, 2006.

11. On en trouvera une exposition très claire dans le livre de L. Malson, Les enfants sauvages: mythe et réalité, Paris, 10/18, 1964.

12. Sur le rapport du sens au non-sens, voir G. Deleuze, Logique du sens, Paris, Minuit, 1969.

13. Bien que dans un autre cadre, celui de la folie, de sa détermination et de son "dépistage ", voir M. Mannoni, Éducation impossible, Paris Seuil, 1973, p. 11 : « Il arrive ainsi que l'on porte sur un enfant de trois ans un diagnostic qui fige les parents dans une attitude d'impuissance - et enferme l'enfant dans un destin institutionnel ».

14. Pour une critique de ces rapports, et du rapport « caritatif » ou " amical » en particulier, on lira F. Deligny, par exemple Graine de crapule, dans CEuvres, Paris, L'Arachnéen, 2007, p. 123-124. Morceaux choisis : «Tu es trop sévère? Ils vont se cacher. Tu ne l'es pas assez? Alors tu ne les empêches pas de mal faire. Ne te soucie donc pas de sévérité.»; «Petits malchanceux ? Voire. Laisse les bonnes âmes des bonnes œuvres se chatouiller la sensiblerie. Toi, fais ton métier. »; «Ils connaissent toutes les méthodes de séduction, de la main sur l'épaule au coup de pied quelque part en passant par le sermon à voix contenue, les yeux dans les yeux. Pour l'effet que ça leur a fait, essaie autre chose. »; "Il faut savoir ce que tu veux. Si c'est te faire aimer d'eux, apporte des bonbons. Mais le jour où tu viendras les mains vides, ils te traiteront de grand dégueulasse. Si tu veux faire ton travail, apporte leur une corde à tirer, du bois à casser, des sacs à porter. L'amour viendra ensuite, et là n'est pas ta récompense. » 
15. L'école fut ouverte en 2008. Elle a fermé ses portes à l'automne 2011. Les bases de ce texte ont été rédigées quelques mois avant la fermeture de l'école : d'où l'emploi du temps présent. Mais le projet d'une analyse critique théorique et pratique de l'éducation se redéploie maintenant sur d'autres voies d'expérimentation, et Pédagogie Nomade continue, bien que sous d'autres formes. On trouvera une présentation de l'école, textes, réflexions, articles de presses, ainsi que des nouveaux projets en cours sur le site www.peripleenlademeure.com.

16. L'ensemble de cette lecture repose sur les hypothèses développées par Etienne Balibar dans son livre Violence et civilité, Paris, Galilée, 2010, sans lequel elle ne serait pas possible : j'y renvoie donc le lecteur avec insistance.

17. L'expression est d'O. Mannoni, dans «Itard et son sauvage », Clefs pour l'imaginaire ou l'autre scène, Paris, Seuil, 1965, coll. Points, 1985.

18. À ce sujet, voir les Réflexions sur l'éducation de Kant, par exemple, trad. A. Philonenko, Paris, Vrin, ainsi que l'Idée d'une histoire universelle d'un point de vue cosmopolitique (et le commentaire d'André Tosel dans Kant révolutionnaire : droit et politique, Paris, PUF, Philosophies, 1988). Sur un plan métapsychologique, dans une perspective plus dialectique, on se reportera à la psychanalyse freudienne bien sûr, mais aussi, élargie au problème de la culture et de la civilisation, à l'entreprise célèbre de Norbert Elias dans La civilisation des mœurs (1969), Paris, Calmann-Lévy, 1973, rééd. Pocket, 2003.

19. Voir, sur ce dernier point, les travaux de M. Mannoni, en particulier Éducation impossible, Paris, Seuil, 1973, rééd. 2008 et Un lieu pour vivre : les enfants de Bonneuil, leurs parents, et l'équipe des soignants, Paris, Seuil, 1976 ; plus généralement, on se reportera au classique Chr. Baudelot et R. Establet, L'école capitaliste en France, Paris, Maspero, 1972, et aux travaux de Bourdieu et Passeron, mais aussi au texte des enfants de Barbiana qui met remarquablement en évidence ces différents procédés, Lettera a una professoressa, dans la très belle traduction parue aux éditions du Chien Noir, Limerlé (Gouvy), 2010.

20. J. Rancière, Le maître ignorant, Paris, Fayard, 1987, rééd. 10/18.

21. Je reprends ici des analyses essentielles développées par Grégory Cormann dans un tout autre cadre, sur un autre sujet, à savoir les grèves de la faim et la séquestration des patrons dans les années 1970: «Existence, techniques du corps et violence chez Sartre. Esquisses pour une anthropologie politique des émotions », H. Feger \& M. Hackel (éd.), Existential Philosophy and Ethics , Berlin, De Gruyter, 2012 ; voir aussi G. Cormann et J. Hamers, « ‘Ce qu'il est con...' Des idées aux corps : Sartre, Baader et les grèves de la faim » dans Les Temps Modernes, $\mathrm{n}^{\circ}$ 667, Paris, Gallimard, janvier-février 2012.

\section{RÉSUMÉS}

Ces deux textes ont été écrit pendant les trois années d'ouverture d'une école différente en Communauté française de Belgique, Pédagogie Nomade. Ils constituent à la fois un témoignage et une tentative de problématisation: problématisation de cette expérience pédagogique, de ses apports, de ses difficultés, voire de ses impasses et de ses échecs; problématisation, plus largement, des questions d'éducation que Pédagogie Nomade a permis d'interroger, sinon à nouveaux frais, du moins d'un regard neuf. 1 . Sens et non-sens à l'école. On dit souvent que l'école souffre l'absence de sens. Qu'il lui faudrait retrouver des apprentissages qui aient du sens; des rapports entre professeurs et élèves qui aient $d u$ sens; des finalités qui aient $d u$ sens. Les 
quelques lignes qui suivent proposent de prendre le parti inverse, et suggèrent que l'école souffre d'abord du trop de sens dont elle est investie, c'est-à-dire, comme nous allons le voir, de sa difficulté à se rapporter au non-sens qui l'affecte. On prendra Pédagogie Nomade, expérience scolaire menée entre 2008 et 2011 à Limerlé en Ardenne belge6, comme analyseur d'un certain nombre de problèmes éducatifs posés dans l'institution scolaire aujourd'hui, et surtout comme opérateur de leur déplacement et de leur reformulation. 2. Violence, éducation, civilité: Pédagogie Nomade analyseur de l'institution scolaire. Ce texte cherche à montrer que Pédagogie Nomade, expérimentation scolaire en Communauté française fondée sur l'idée d'égalité entre professeur et élèves et appuyée sur un principe de cogestion, prenant à rebours le mythe moderne de l'éducation, tente de formuler à nouveaux frais les problèmes posés par la violence dans le rapport pédagogique tel qu'il est structuré dans l'institution scolaire.

\section{INDEX}

Thèmes : anthropologie, philosophie sociale, philosophie politique, éthique, philosophie de l'éducation

Index géographique : Belgique

Index chronologique : XXe siècle

Mots-clés : éducation, école, violence, égalité, Pédagogie Nomade

\section{AUTEUR}

\section{ANTOINE JANVIER}

Antoine Janvier est collaborateur scientifique du FRS-FNRS au département de philosophie de l'ULg. Ses travaux portent sur la philosophie de Gilles Deleuze, ainsi que sur la philosophie morale et politique contemporaine, en particulier sur les questions d'éducation.

Antoine.Janvier@ulg.ac.be 